\title{
Consensus formation on a triad scale-free network
}

\author{
A.O. Sousa ${ }^{1}$ \\ Institute for Computer Physics (ICP), University of Stuttgart, \\ Pfaffenwaldring 27, 70569 Stuttgart, Germany.
}

\begin{abstract}
Several cases of the Sznajd model of socio-physics, that only a group of people sharing the same opinion can convince their neighbors, have been simulated on a more realistic network with a stronger clustering. In addition, many opinions, instead of usually only two, and a convincing probability have been also considered. Finally, with minor changes we obtain a vote distribution in good agreement with reality.
\end{abstract}

Key words: Social systems, Scale-free networks, Small-world systems.

PACS: 89.65.-s, 89.75.-k, 05.10.-a

\section{Introduction}

A growing interest has focused on the statistical physics of complex networks. They describe a wide range of systems in nature and society, modeling diverse systems as the world wide web, the net of human sexual contacts, or a network of chemicals linked by chemical reactions [1]. Such networks posse a rich set of scaling properties. A number of them are scale-free, that is, the probability that a randomly selected node has exactly $k$ links decay as a power law, following $P(k) \sim k^{-\gamma}$, where $\gamma$ is the degree exponent, in consequence they present resilience against random breakdowns, this effect is known as robustness of the network. The shortest-path-length between their sites grows slowly (i.e. logarithmically) with the size of the network. That is, in spite of large sizes of networks, the distance between their sites are short - a feature known as the "small world" effect.

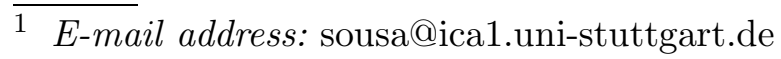



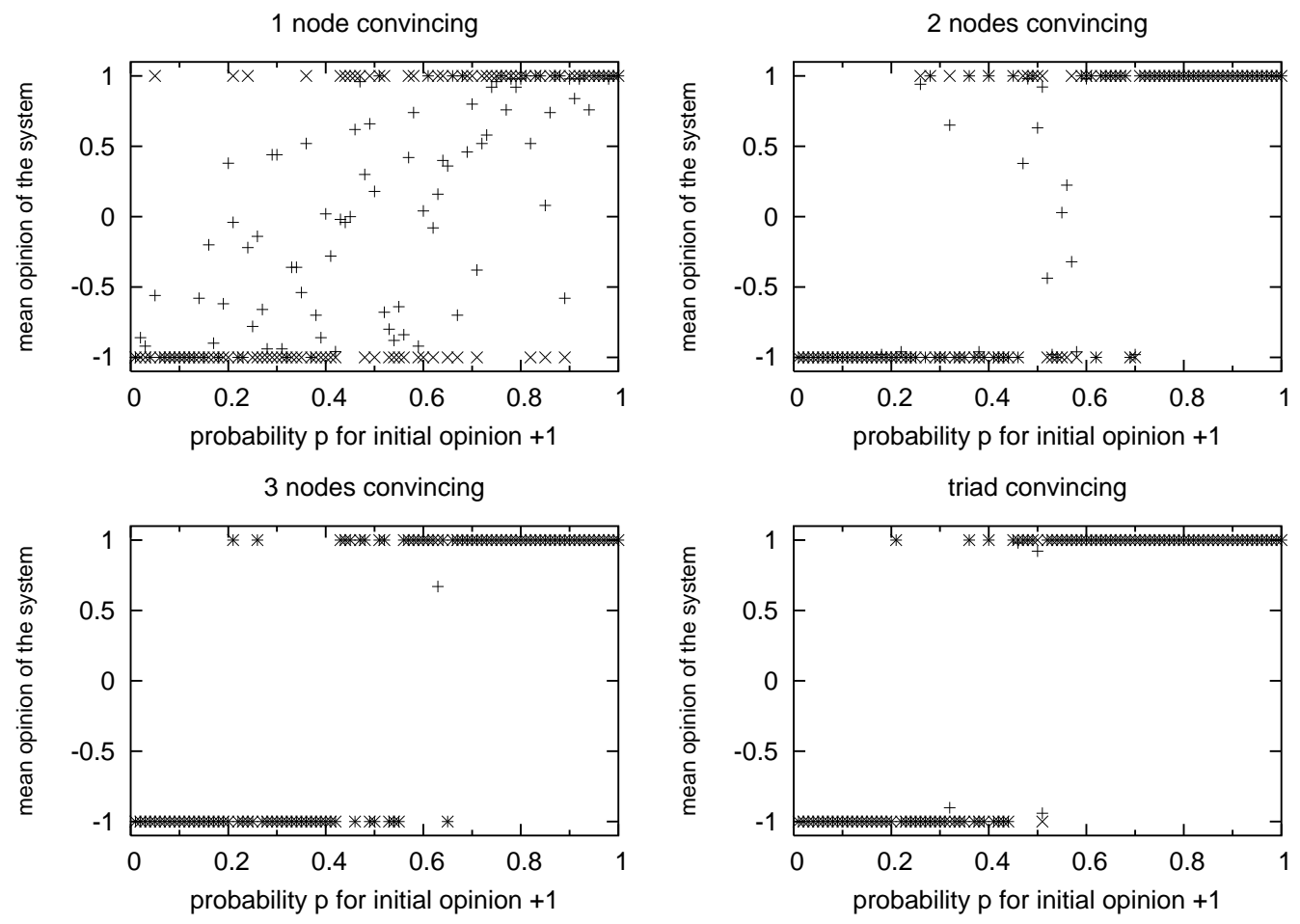

Fig. 1. Mean opinion of the system for 1 node (on top left), 2 nodes (on top right), 3 nodes (on bottom left) and triad (on bottom right) convincing, and different initial concentrations $p$ of opinion +1 after $t=1(+)$ and $t=10(\mathrm{x})$ timesteps.

The majority of networks used to generate a scale-free topology are stochastic, they create networks in which the nodes appear to be randomly connected to each other. Scale-free random networks have naturally a continuous degree distribution spectrum, but recently it has been shown that discrete degree distributions of some deterministic graphs also have a power law decay [1]. Furthermore, scale-free random networks are excellently modeled by such deterministic graphs [2]. However the comparison between the behavior of stochastic and deterministic networks in the simulation of a particular model still remains open.

The involvement of physics in research on spreading of opinion's models has been a matter of increasing studies nowadays (see [3] for a review). Of particular interest here is the Sznajd model [4], which is one of several recent consensus-finding models [5] and in which each randomly selected pair of nearest neighbors convinces all its neighbors of the pair opinion, only if the pair shares the same opinion; otherwise, the neighbor opinions are not affected. One time step means that on average every lattice node is selected once as the first member of the pair. It differs from other consensus models by dealing only with communication between neighbors, and the information flows outward, as in rumors spreading. In contrast, in most other models the information flows inward. Initially, two opinions $( \pm 1)$ are randomly distributed 
with probability $p$ over all the nodes of the lattice. The basic Sznajd model with random sequential updating always leads to a consensus (all sites have opinion +1 or -1 and the whole system reaches a fixed point after a certain time of simulation). A phase transition is often observed as a function of the initial concentration of opinion $p$. A generalization to many different opinions (instead of only \pm 1 ) put into a Barabási-Albert network [1] reproduced quite well the results of the complex elections of city councillors in the state of Minas Gerais in Brazil [6].

\section{The model}

\subsection{The triad network}

Although the Barabási-Albert [1] network has successfully explained the scalefree nature of many networks, a striking discrepancy between it and real networks is that the value of the clustering coefficient - which is the probability that two nearest neighbors of the same node are also mutual neighbors - predicted by the theoretical model decays very fast with the network size and for large systems is typically several orders of magnitude lower than found empirically (it vanishes in the thermodynamic limit $[7,10]$ ). In social networks, for instance, the clustering coefficient distribution $C(k)$ exhibits a power-law behavior, $C(k) \propto k^{-\gamma}$, where $k$ is number of neighbors (degree or connectivity) of the node and $\gamma \approx 1$ (everyone in the network knows each other).

Very recently, by adding a triad formation step on the Barabási-Albert prescription, this problem has been surmounted and scale-free models with high clustering coefficient have been investigated [7]. The Barabási-Albert network starts with a small number $m(m=3$ in our simulations) of sites (agents, people) all connected with each other. (For $m=2,4$ and 5 we observed similar results for 100 runs each.) Then a large number $N$ of additional sites is added as follows: First, each new node (node $i$ ) performs a preferential attachment step, i.e, it is attached randomly to one of the existing nodes (node $j$ ) with a probability proportional to its degree; then follows a triad formation step with a probability $p_{\mathrm{tf}}$ : the new node $i$ selects at random a node in the neighborhood of the one linked to in the previous preferential attachment step (node $j$ ). If all neighbors of $j$ are already connected to $i$, then a preferential attachment step is performed ("friends of friends get friends"). In this model, the original Barabási-Albert network corresponds to the case of $p_{\mathrm{tf}}=0$. It is expected that a nonzero $p_{\text {tf }}$ gives a finite nonzero clustering coefficient as $N$ is increased, while the clustering coefficient goes to zero when $p_{\mathrm{tf}}=0$ (the BA scale-free network model). 


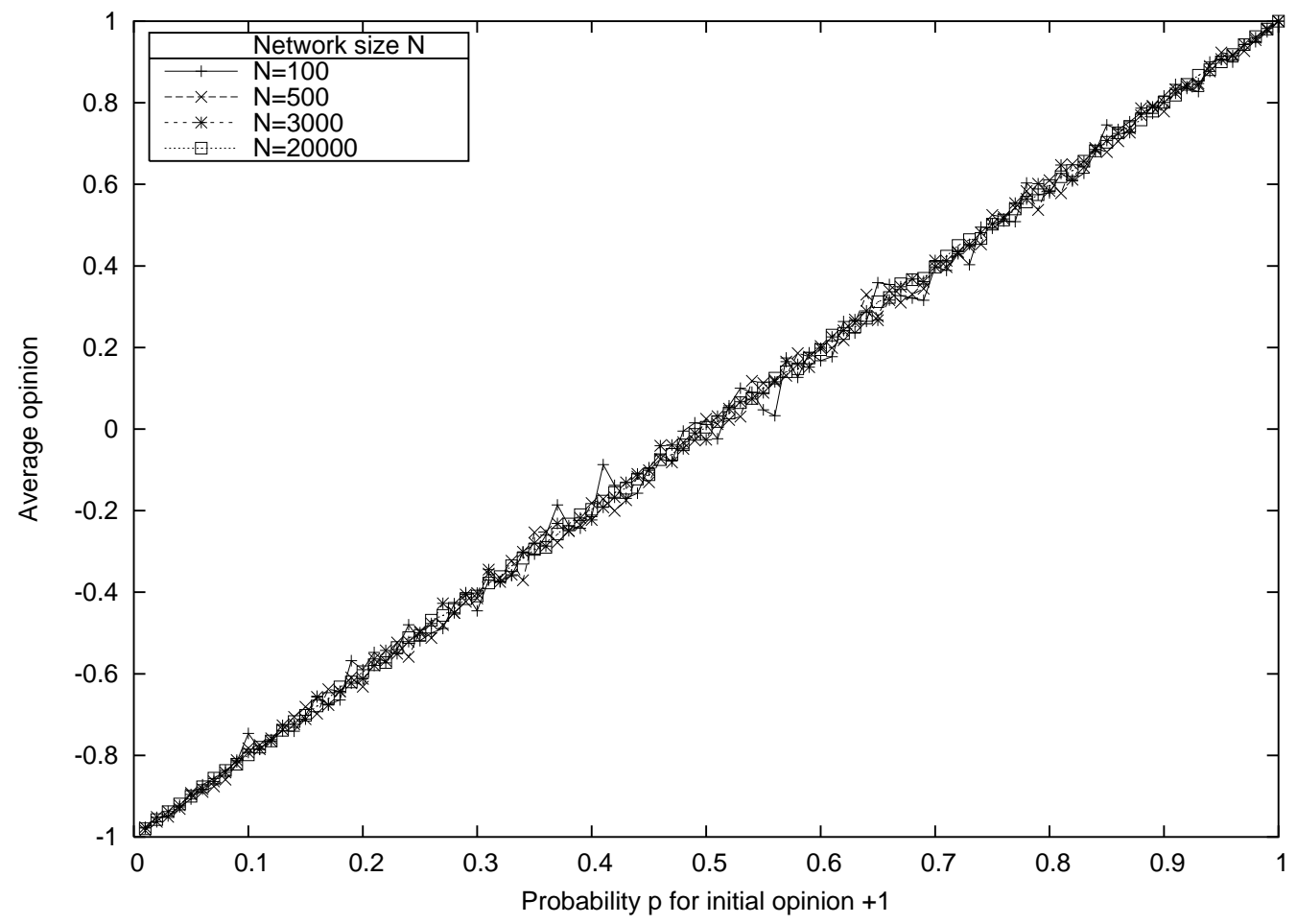

Fig. 2. The average opinion versus the probability $p$ for initial opinion +1 , when 1 node convincing strategy is considered for different network size $N: N=100(+)$, $N=500(\times), N=3000(*)$ and $N=20000(\bullet)$.

\subsection{The Sznajd model}

\subsection{Binary opinions}

Every node in the network is considered to be an individual with an opinion that in the beginning of the simulation is randomly chosen with probability $p$ for opinion +1 . Once the network has been completely constructed, we start the consensus process of Sznajd. At every time step $t>0$, all the nodes are randomly visited and updated (a random list of nodes assures that each node is reached exactly once) by following four variations:

- 1 site convincing: For each site $i$ chosen, we change the opinion of all its neighbors to the site's opinion.

- 2 sites convincing: For each site $i$ chosen, we select randomly one of its neighbors. If this selected neighbor has the same opinion as the site $i$, then all their neighbors follow the pair's opinion. Otherwise, nothing is done.

- 3 sites convincing: For each $i$ site chosen, we select 2 of its neighbors at random. If all these three sites have the same opinion, they change the opinion of all their neighbors. 


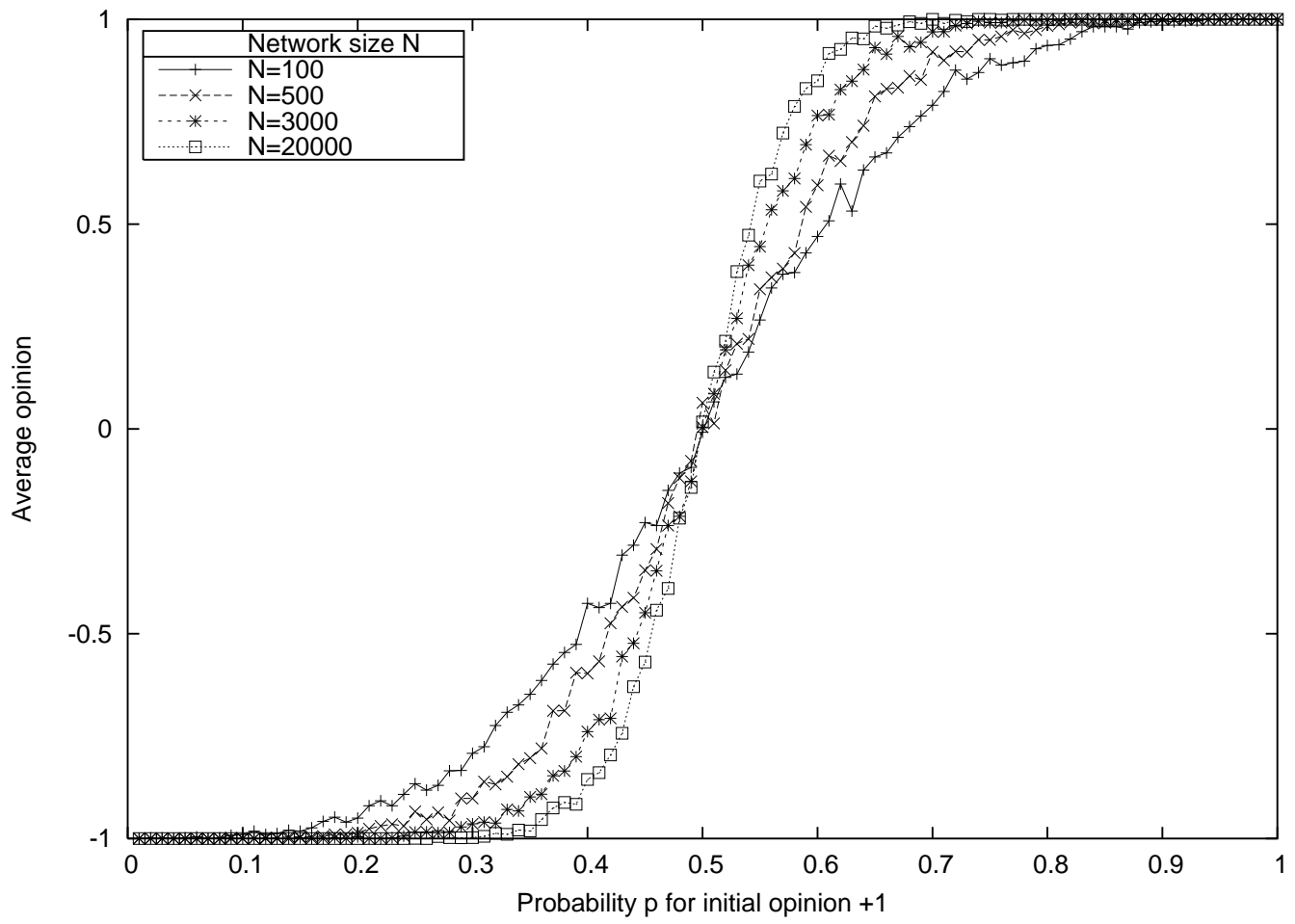

Fig. 3. As Fig. 2, but for the 2 nodes convincing case.

- triad convincing: For each $i$ site chosen, we select 2 of its neighbors at random. If all these three sites form a triangle and have the same opinion, they change the opinion of all their neighbors. In the previous case, the 3 nodes must be connected with each other, they must not necessarily form a triangle.

In Figure (1) we present the mean opinion of the network as a function of the initial concentration $p$ of opinion +1 for all the cases mentioned above after $t=1$ and $t=10$ timesteps. From this figure we can see that after $t=1$ timestep in all the cases a full consensus cannot be reached, however after $t=$ 10 timesteps the whole system always lead to a fixed point: consensus (all sites have opinion +1 or -1 ). Figures 2,3 and 4 correspond to the average of the results obtained for 1000 samples (1000 different initial seeds for the random number generator). For 2 nodes, 3 nodes and triad (not shown) convincing rules one can observe a phase transition: concentration $p>1 / 2$ lead to full consensus +1 and concentrations $p<1 / 2$ to full consensus -1 for large enough systems. Since in a finite network, of course, phase transitions are never sharp, and thus the transition is indicated numerically by a slope (Figs. 3 and 4) becoming the steeper the larger the network size is. In an infinite network, one would get a sharp step function for the mean opinion of the sytems versus the initial concentration $p$ of opinion +1 . This does not hold for the 1 node convincing case (see Fig. 2) where there is no such transition and the mean opinion of the system varies smoothly as a function of the initial density of 


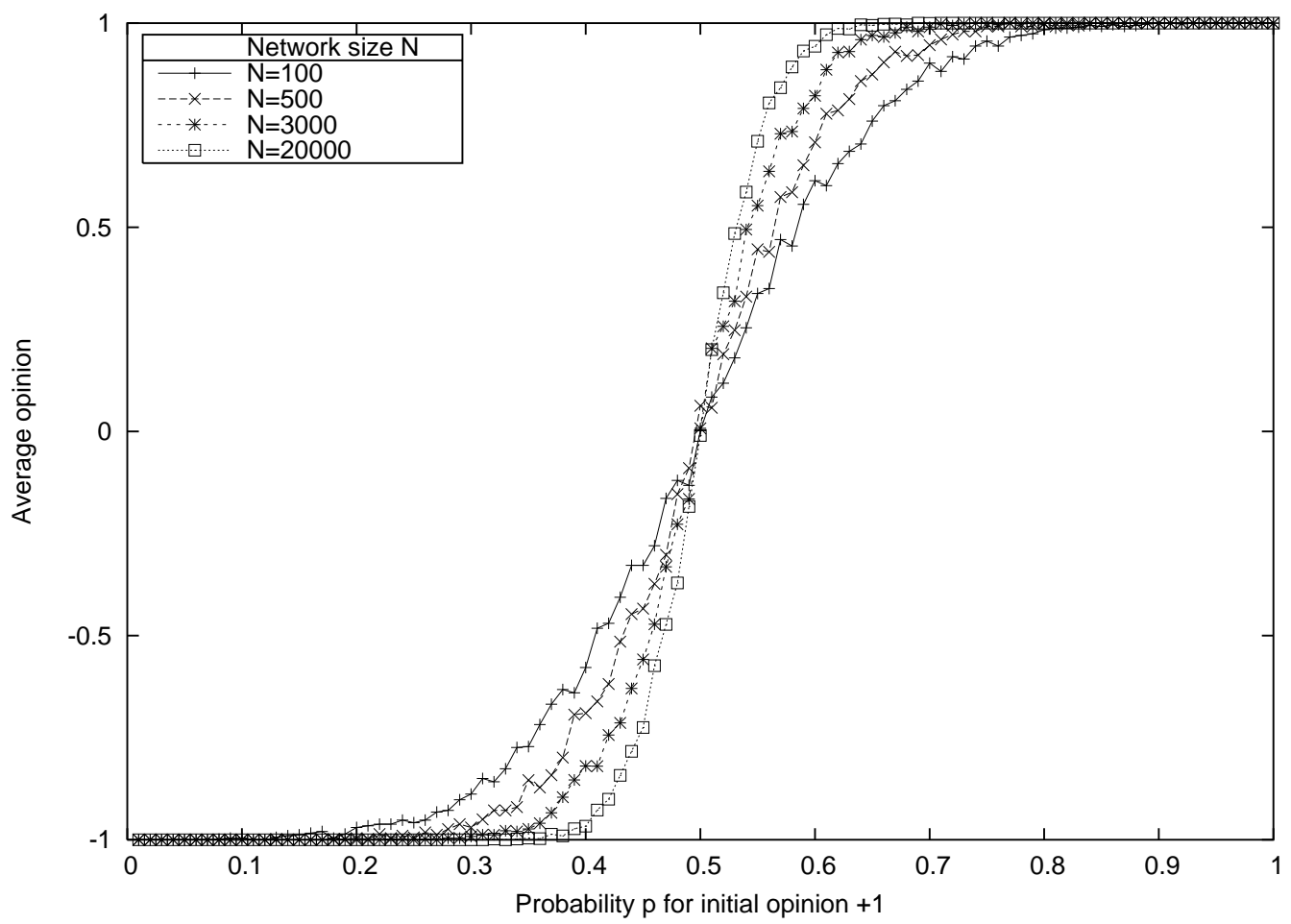

Fig. 4. As Fig. 2, but for the 3 nodes convincing case.

opinion +1 , as well the results does not have any dependence on the network size $N$. The phase transition at $p_{c}=1 / 2$ here observed does not exist in one dimension [4] or when a single site [9] (instead of a pair or plaquette) on the square lattice [8] convinces its neighbors, although it is also found on the square lattice when a plaquette or a neighboring pair persuades its neighbors [8], on a correlated-diluted square lattice [11], on a triangular and simple cubic lattice if a pair convinces its 8 (or 10, respectively) neighbors [13], on the Barabási-Albert network [12] and on a pseudo-fractal network [14].

\subsection{Multi-pluralist world}

Instead of beginning the simulation with only two opinions, $S_{i}=+1$ or $S_{i}=-1$, now we analyze the model when many different opinions are considered. After generating the triad network, as explained before, every individual assumes an opinion randomly selected from $N_{c}$ different opinions, with $N_{c}$ ranging from 2 opinions $\left(S_{i}=+1\right.$ or $\left.S_{i}=-1\right)$ to the network size $N\left(S_{i}=1\right.$ or $S_{i}=2$ or $\left.S_{i}=3 \ldots S_{i}=N\right)$ and $i=[1,2,3, \ldots, N]$. At every time step $t>0$, all the nodes are randomly selected and updated by following the rules previously described.

In Figures 5 and 6 we present the fraction of samples, out of 1000, ending with 


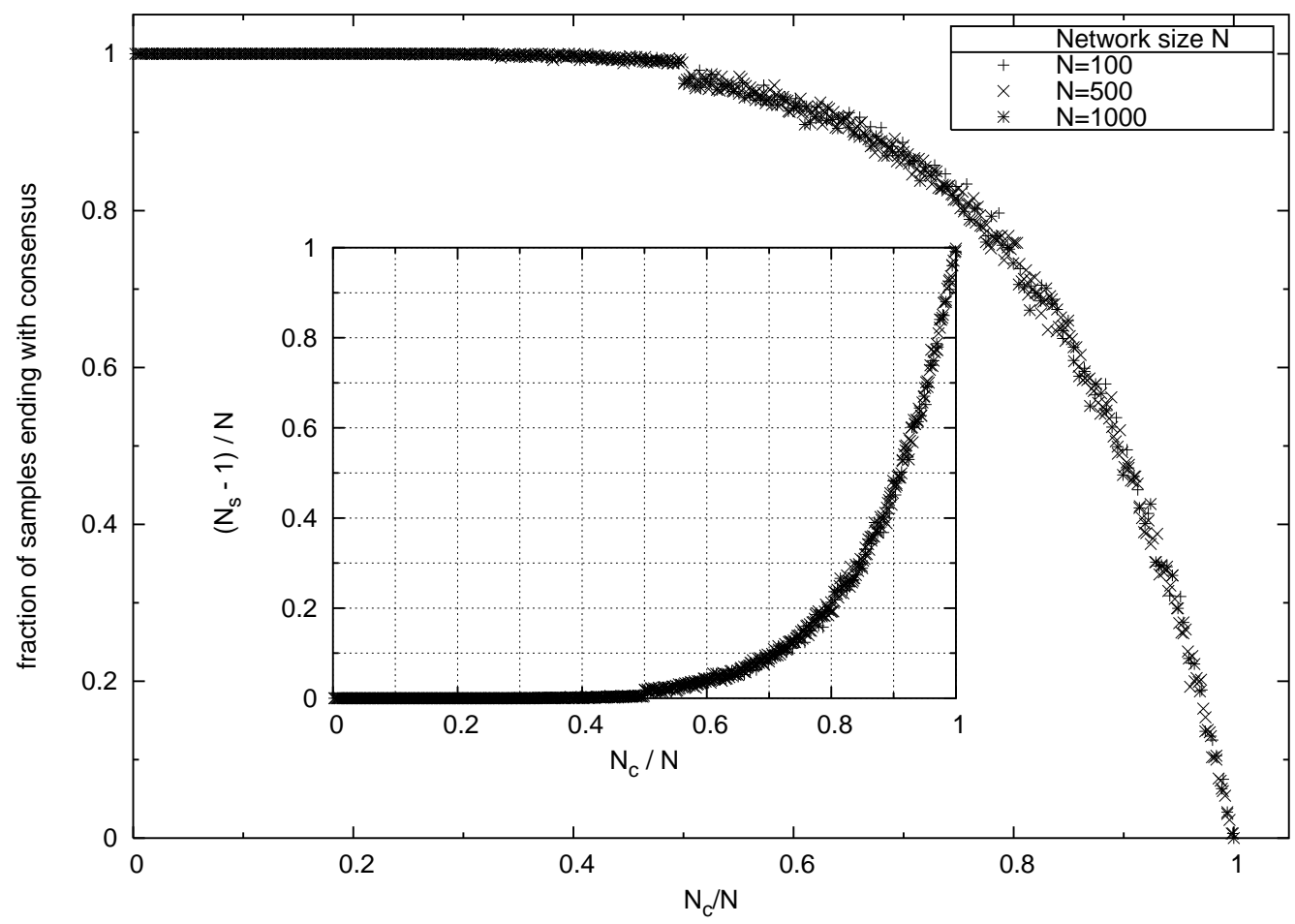

Fig. 5. Consensus fraction and scaled final number of different opinions $\left(\left(N_{s}-1\right) / N\right)$ versus the scaled number of different ones at the beginning of the simulation $\left(N_{c} / N\right)$ for the 2 nodes convincing rule and when all sites have a random initial opinion.

full consensus as a function of the scaled number $N_{c} / N$ of different opinions at the beginning of the simulation. The insets show the scaled number $N_{s}$ of different opinions at the end of the simulation versus the scaled number $N_{c}$ of different ones at the beginning of the simulation. The 1 node convincing rule (not shown) always leads the system to a full consensus (the whole system reaches a fixed point after some time-steps, i.e., all sites have the same opinion) independently of the initial number of different opinions and the network size $N$. Nevertheless, if the system evolves under the 2 nodes convincing rule (Figure 5), all the samples reach a fixed point with all the individuals having the same opinion $\left(N_{s}=1\right.$, see inset) when $N_{c}<0.50$; and for $N_{c}>0.50$ the fraction of samples which has reached a full consensus decays slowly towards to zero as the scaled initial number $N_{c}$ of different opinions increases, as well the number $N_{s}$ of surviving opinions grows exponentially with $N_{c}$ and independent of $N$. If the 3 nodes or triad (not shown) convincing rule is considered (see Fig. 6), only when $N_{c}<0.1$ the complete consensus $\left(N_{s}=1\right.$, see inset) is found in all the samples, for $0.1<N_{c}<0.5$ the fraction of samples decays slowly as $N_{c}$ increases and $N_{s}$ grows to values closer to $N_{c}$, besides finite size effects appear; when $N_{c}>0.5$ no sample reaches a consensus and $N_{s}$ roughly equals $N_{c}$, that means everybody keeps its own opinion and a fixed point is reached soon. 


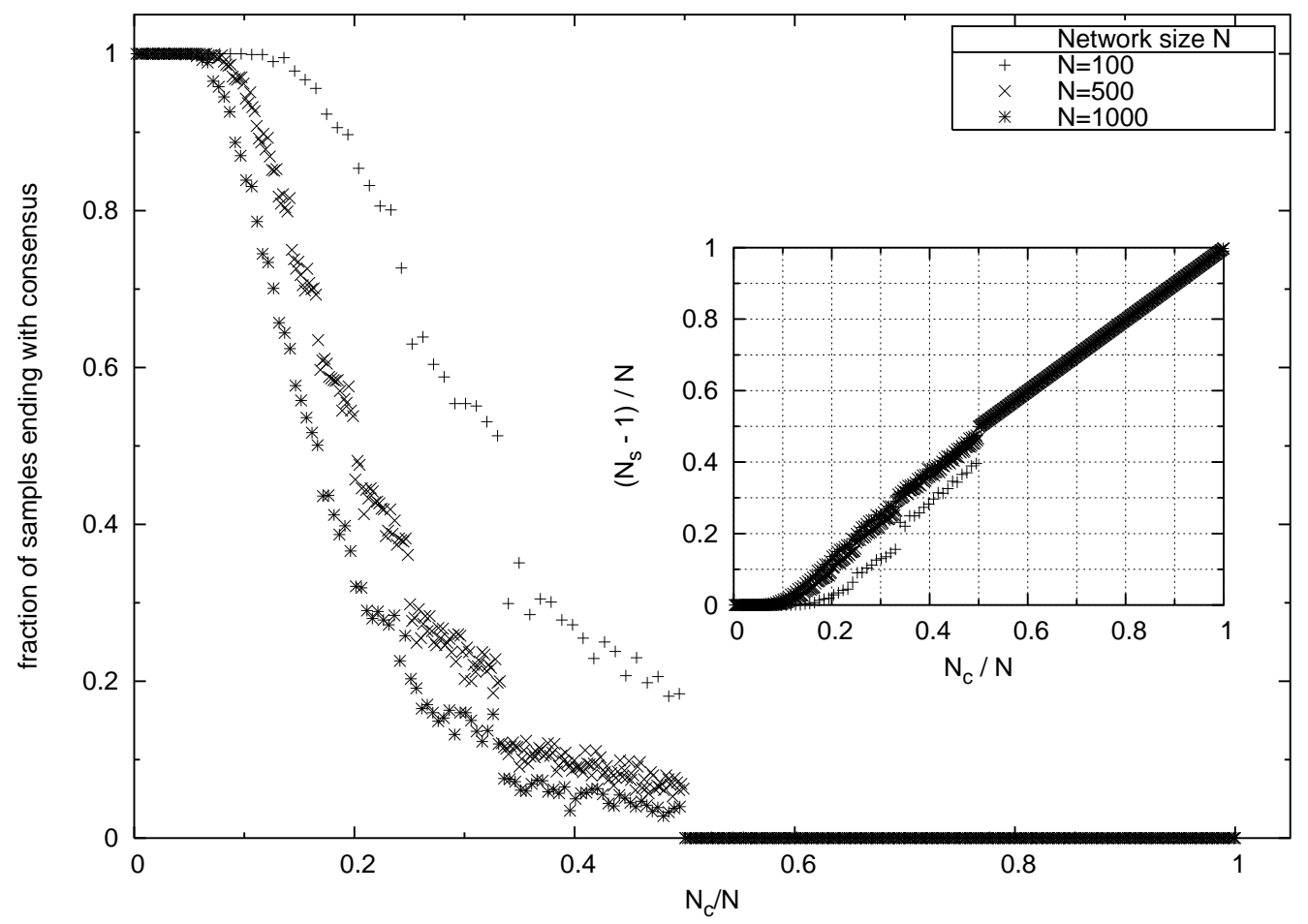

Fig. 6. As Fig. 5, but for the 3 nodes convincing rule.

Additionally, we have also simulated the 2 nodes convincing rule when only a fraction $N_{c}$ of individuals at the beginning of the simulation assumes an opinion. In contrast to the same rule analyzed above where the simulation starts with all the individuals having an opinion, in such way if $N_{c}<N$ some individuals can assume the same opinion, now $N-N_{c}$ individuals do not assume any opinion $\left(S_{i}=0\right)$ and they are convinced to follow the neighbor's opinion according to probability $p_{c}$ : a pair of neighbors in agreement convinces all the nodes connected with them with a probability for each of the two nodes inversely proportional to the time-independent number $k$ of nodes connected to it, $p_{c}=k^{-1}$. Alternatively, this case can be associated as an election campaign when $N_{c}$ candidates try to persuade $N$ voters to vote for them. As in real elections, we do not wait for a kind of equilibrium state, but count the votes at some intermediate time.

Figure 7 shows the distribution of the number of candidates getting $v$ votes each at two different timesteps $t=15(+)$ and $t=20(\times)$. In agreement with findings for real elections which shows that the distribution of the number of candidates $N_{v}$ receiving a fraction of votes $v$ follows a power-law $N_{v} \propto 1 / v$ $[6,14,15]$ except for downward deviations at small and large numbers $v$ of votes, also our simulations obey this hyperbolic law including the deviations at the ends. 


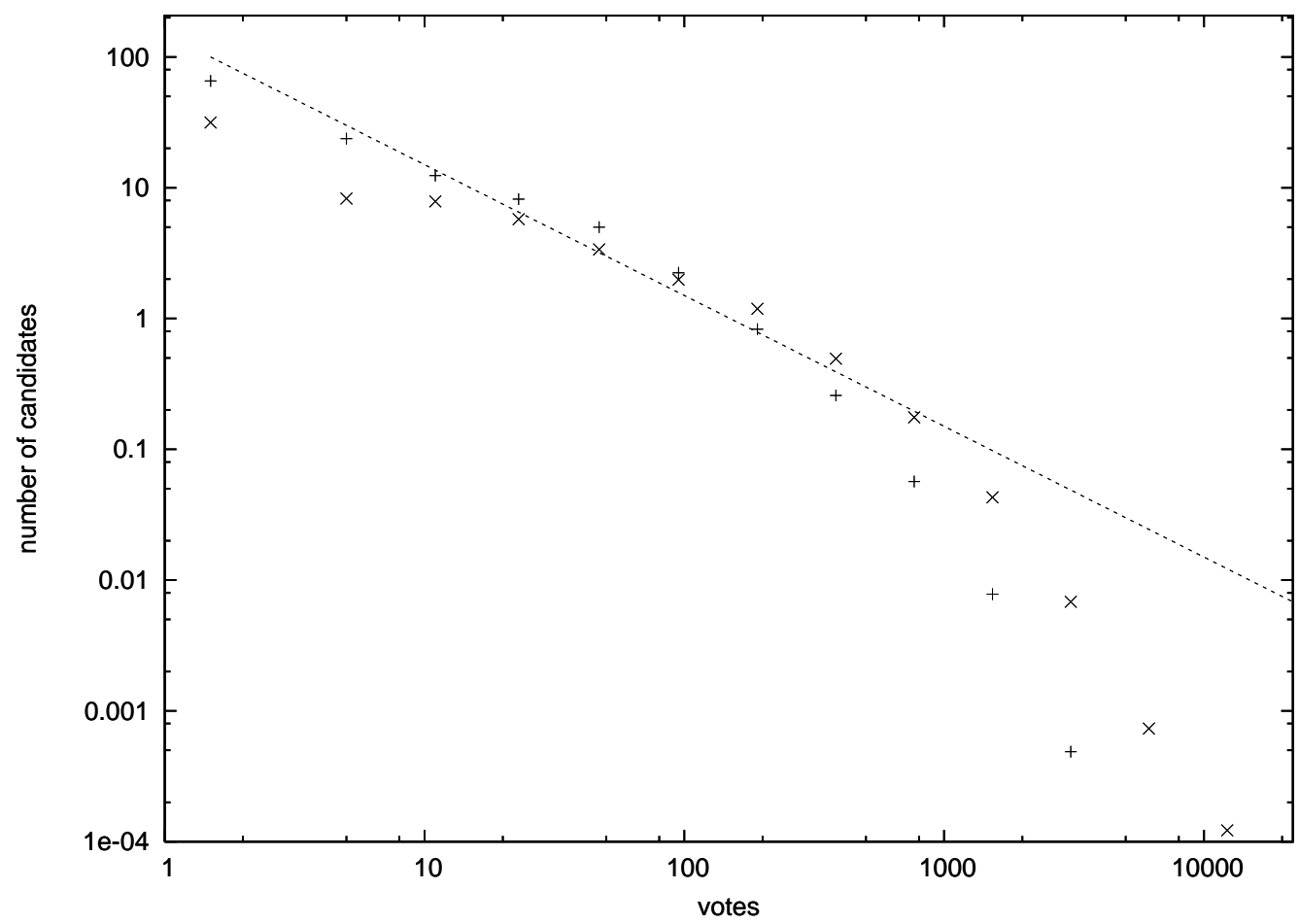

Fig. 7. Distribution of the number of candidates getting $v$ votes each at two different timesteps $t=15(+)$ and $t=20(\times)$.

\section{Conclusions}

By simulating the Sznajd model of socio-physics on a more realistic network with stronger clustering, we have found a phase transition as a function of the initial concentration of $S=+1$ opinions at $p_{c}=1 / 2$ : For $p<1 / 2$ all samples end up with $S=-1$, and for $p>1 / 2$ they all end up in the other fixed point $S=+1$, for large enough networks. This phase transition exists for 2 nodes, 3 nodes and triad convincing rule, however it does not exist for 1 node convincing rule. Instead of only two opinions, but considering many opinions, we notice that 1 node convincing rule leads the system always to a complete consensus, while for the 2 nodes convincing rule a full consensus is obtained when $N_{c} / N<0.5$ and if $N_{c} / N>0.5$ the final number of different opinions grows exponentially with the number $N_{c}$ of initial ones; for 3 nodes and triad convincing rules a full consensus is observed only for $N_{c} / N<0.1$, if $0.1<N_{c}<0.5$ the fraction of samples decays slowly as $N_{c}$ increases and $N_{s}$ grows to values closer to $N_{c}$ and when $N_{c}>0.5$ the final number $N_{s}$ of different opinions roughly equals $N_{c}$. When simple and minor changes are taken into account in the model, the hyperbolic law observed empirically in real elections can be reproduced quite well. 
Acknowledgments: The author thanks D. Stauffer for a critical reading of the manuscript; this work has been supported by a research grant from Alexander von Humboldt.

\section{References}

[1] R. Albert and A.-L. Barabási, Science 286, 509 (1999), A.-L. Barabási, E. Ravasz and T. Vicsek, Physica A 299, 559 (2001); R. Albert and A.-L. Barabási Rev. of Mod. Phys. 74, 47 (2002); A.L. Barabási, Linked: The New Science of Networks, Perseus Publishing, Cambridge MA, 2002.

[2] S.N. Dorogovtsev, A.V. Goltsev and J.F.F. Mendes, Phys.Rev. E 65, 066122 (2002).

[3] D. Stauffer, Journal of Artificial Societies and Social Simulations 5, issue 1, paper 4(jasss.soc.surrey.ac.uk) (2002); Computing in Science and Engineering 5, 71 (May/June 2003); and in The Monte Carlo Method on the Physical Sciences, edited by J. E. Gubernatis, AIP Conference Proceedings, 690, 147 (2003) = cond-mat/0307133

[4] K. Sznajd-Weron and J. Sznajd, Int. J. Mod. Phys. C 11, 1157 (2000).

[5] G. Deffuant, D. Neau, F. Amblard, G. Weisbuch, Adv. Complex Syst. 3, 87 (2000); G. Weisbuch, G. Deffuant, F. Amblard, J.-P. Nadal, Complexity 7, 55 (2002); G. Deffuant, F. Amblard, G. Weisbuch, T. Faure, J. Artificial Soc. Social Simulation (http://jasss.soc.surrey.ac.uk/5/4/1.html); R. Hegselmann and U. Krause, J. Artificial Soc. Social Simulation (http://jasss.soc.surrey.ac.uk/5/3/2.html).

[6] A. T. Bernardes, D. Stauffer, and J. Kertész, Eur. Phys. J. B 25123 (2002).

[7] J. Davidsen, H. Ebel and S. Bornholdt, Phys.Rev.Letters 88, 128701 (2002); P. Holme and B.J. Kim, Phys. Rev. E 65, 026107 (2002); G. Szabó, M. Alava and J. Kertész, Phys. Rev. E 67, 056102 (2003).

[8] D. Stauffer, A.O. Sousa, and S. Moss de Oliveira, Int. J. Mod. Phys. C 11, 1239 (2000).

[9] R. Ochrombel, Int. J. Mod. Phys. C 12, 1091 (2001); F. Slanina and H. Lavicka, Eur. Phys. J. B, 35, 279 (2003).

[10] D. Stauffer, A.O. Sousa, and C. Schulze, to appear in Journal of Artificial Societies and Social Simulation (http://jasss.soc.surrey.ac.uk) (2004) = cond-mat/0310243

[11] A.A. Moreira, J.S. Andrade Jr. and D. Stauffer, Int. J. Mod. Phys. C 12, 39 (2001).

[12] J. Bonnekoh, Int. J. Mod. Phys. C 12, 39 (2003). 
[13] I. Chang, Int. J. Mod. Phys. C 12, 1509 (2001).

[14] M.C. González, A.O. Sousa and H.J. Herrmann, Int. J. Mod. Phys. C 15, 45 (2004).

[15] R.N. Costa Filho, M.P. Almeida, J.S. Andrade Jr. and J.E. Moreira, Phys Rev E 60, 1067 (1999); R.N. Costa Filho, M.P. Almeida, J.E. Moreira and J.S. Andrade Jr., Physica A 322, 276 (2003); H. Situngkir, http://www.ekonofisika.com/bfi/2004k.pdf 